\title{
Thin-section computed tomography findings and longitudinal variations of the residual pulmonary sequelae after discharge in patients with COVID-19: a short-term follow-up study
}

\author{
Die Zhang ${ }^{1} \cdot$ Chen Zhang $^{1} \cdot$ Xiaohe $\mathrm{Li}^{2} \cdot$ Jing Zhao ${ }^{1} \cdot{\text { Chao } \mathrm{An}^{1}}^{1} \cdot$ Cheng Peng ${ }^{1} \cdot$ Lifei Wang $^{1}$ \\ Received: 19 September 2020 / Revised: 23 December 2020 / Accepted: 16 February 2021 / Published online: 11 March 2021 \\ (C) European Society of Radiology 2021
}

\begin{abstract}
Objectives This study analyzed and compared CT findings and longitudinal variations after discharge between severe and nonsevere coronavirus disease (COVID-19) patients who had residual pulmonary sequelae at pre-discharge.

Methods A total of 310 patients were included and stratified into severe and non-severe COVID-19 groups. Cross-sectional CT features across different time periods (T0: pre-discharge, T1: 1-4 weeks after discharge, T2: 5-8 weeks after discharge, T3: 9-12 weeks after discharge, T4: > 12 weeks after discharge) were compared, and the longitudinal variations of CT findings were analyzed and compared in both groups.

Results The cumulative absorption rate of fibrosis-like findings in the severe and non-severe groups at T4 was $24.3 \%$ (17/70) and $52.0 \%$ (53/102), respectively. In both groups, ground-glass opacity (GGO) with consolidation showed a clear decreasing trend at $\mathrm{T} 1$, after which they maintained similar lower levels. The GGO in the severe group showed an increasing trend first at T1 and then decreasing at T4; however, the incidence decreased gradually in the non-severe group. Most fibrosis-like findings showed a tendency to decrease rapidly and then remained stable. Bronchial dilatation in the severe group persisted at an intermediate level. Conclusions After discharge, the characteristics and changing trends of pulmonary sequelae caused by COVID-19 were significantly different between the two groups. Pulmonary sequelae were more serious and recovery was slower in patients with severe/ critical disease than in patients with moderate disease. A portion of the fibrosis-like findings were completely absorbed in patients with moderate and severe/critical diseases.

Key Points

- Lung sequelae were more serious and recovery was slower in severe/critical COVID-19 patients.

- Complete absorption of fibrosis-like findings after a short-term follow-up was observed in at least 17/70 (24.3\%) of COVID-19 patients with severe/critical disease and 53/102 (52.0\%) of COVID-19 patients with moderate disease.

- The most common fibrosis-like findings was a parenchymal band; irregular interface was a nonspecific sign of COVID-19, and the percentage of bronchial dilatation in patients with severe/critical disease remained at a relatively stable medium level (range, 31.6 to $47.8 \%$ ) at all stages.
\end{abstract}

Keywords Coronavirus $\cdot$ Pulmonary fibrosis $\cdot$ Lung injury $\cdot$ Multidetector computed tomography

Die Zhang and Chen Zhang contributed equally to this work.

Lifei Wang

wanglf007n@163.com

1 Department of Radiology, National Clinical Research Center for Infectious Disease, Shenzhen Third People's Hospital, The Second Affiliated Hospital, School of Medicine Southern University of Science and Technology, 29 Bulan Road, Longgang District, Shenzhen 518000, Guangdong, China
2 Department of Infectious Disease, National Clinical Research Center for Infectious Disease, Shenzhen Third People's Hospital, The Second Affiliated Hospital, School of Medicine Southern University of Science and Technology, 29 Bulan Road, Longgang District, Shenzhen 518000, Guangdong, China 


\section{Abbreviations}

CO Consolidation

COVID-19 Coronavirus disease

GGO Ground-glass opacity

pGGO Pure ground-glass opacity

SARS-CoV-2 Severe acute respiratory syndrome coronavirus 2

\section{Introduction}

Coronavirus disease (COVID-19), caused by severe acute respiratory syndrome coronavirus 2 (SARS-CoV-2) infection, has been spreading worldwide since December 2019 [1, 2]. The virus is characterized by rapid spreading and strong infectivity. As of August 10, 2020, the disease has caused at least 728,013 deaths and 19,718,030 infections worldwide, and the numbers continue to increase [3]. However, COVID-19 mortality is approximately $3.7 \%$, which is lower than that of SARS (9.6\%) and Middle East respiratory syndrome $(34.4 \%)[3,4]$. These numbers suggest that there should be a large number of survivors from COVID-19 after the pandemic. Thus, follow-up management after discharge is an important issue for both the public and the medical community.

Follow-up management of viral pneumonia is a key factor in preventing re-transmission after discharge [5]. According to previous studies, more than $80 \%$ of patients with COVID-19 have residual pulmonary damage at the time of hospital discharge $[6,7]$. Additionally, pulmonary sequelae, including ground-glass opacity (GGO), consolidation (CO), and fibrosis, are present in patients after discharge [8-10]. However, these previous studies had a follow-up time $<1$ month, and thus, it is not clear whether lung injury persists long-term [8-10]. Moreover, there have been few reports about changes in lung damage after discharge in patients with severe and critical COVID-19. A better understanding of the longitudinal changes in CT manifestations in COVID-19 patients with residual pulmonary sequelae at discharge is a significant value for post-discharge management and prognosis monitoring, and, more importantly, can provide insights into the degree of lung injury and repair.

Therefore, this study retrospectively analyzed and compared the differences and longitudinal variations of pulmonary sequelae caused by COVID-19 between moderate and severe/ critical patients during a short-term follow-up, using a series of CT scans.

\section{Materials and methods}

The ethics committee of our hospital approved the study and waived the requirement for obtaining informed consent from patients for this retrospective study. However, informed consent was obtained from all patients to perform the CT examination.

\section{Patients}

From January 11 to July 5, 2020, 459 patients were diagnosed with SARS-CoV-2 infection by reverse transcriptionpolymerase chain reaction (RT-PCR) and all met the discharge standards after treatment [11]. Diagnoses were made according to the Chinese guidelines [11]. Detailed diagnostic and discharge criteria are presented in the supplementary materials.

Exclusion criteria were as follows: (1) mild cases; (2) CT scans not performed after discharge; (3) complete absorption of lesions on radiological images before discharge; (4) $>7$ days had elapsed from the last pre-discharge CT scan to discharge; and (5) serious complications requiring long-term bed rest (e.g., intracerebral hemorrhage).

Finally, 310 patients were included (Fig. 1a) and divided into two groups according to clinical classification: severe (severe and critical cases) and non-severe (moderate cases). Clinical data (sex, age, length of hospitalization, days from discharge to follow-up CT) were collected. Common underlying diseases, including emphysema, chronic bronchitis, advanced interstitial lung disease, obsolete pulmonary tuberculosis, hypertension, diabetes, and coronary disease, were also recorded.

\section{Severity classification}

Disease severity was graded according to the Chinese guidelines [11]. The patients were classified as having (1) mild disease: mild symptoms without imaging manifestations of pneumonia; (2) moderate disease: fever, respiratory manifestations, and radiological findings indicating pneumonia; (3) severe disease: presenting any of the following: (i) respiratory rate $\geq 30 / \mathrm{min}$; (ii) resting oxygen saturation $\leq 93 \%$; (iii) arterial oxygen partial pressure $\left(\mathrm{PaO}_{2}\right)$ /fraction of inspired oxygen $\left(\mathrm{FiO}_{2}\right) \leq 300 \mathrm{mmHg}$ $(1 \mathrm{mmHg}=0.133 \mathrm{kPa}$ ); or (iv) progression in $>50 \%$ of lesions within 24 to $48 \mathrm{~h}$ on lung imaging; and (4) critical disease: presenting any of the following: (i) respiratory failure requiring mechanical ventilation; (ii) shock; or (iii) failure of other organs requiring admission to the intensive care unit.

\section{CT follow-up strategy}

After discharge, each patient underwent follow-up plain CT assessments based on clinical scenario; the CT follow-up ended when the pulmonary lesions were completely absorbed or continued until the end of the study (July 5, 2020). Five time 
a

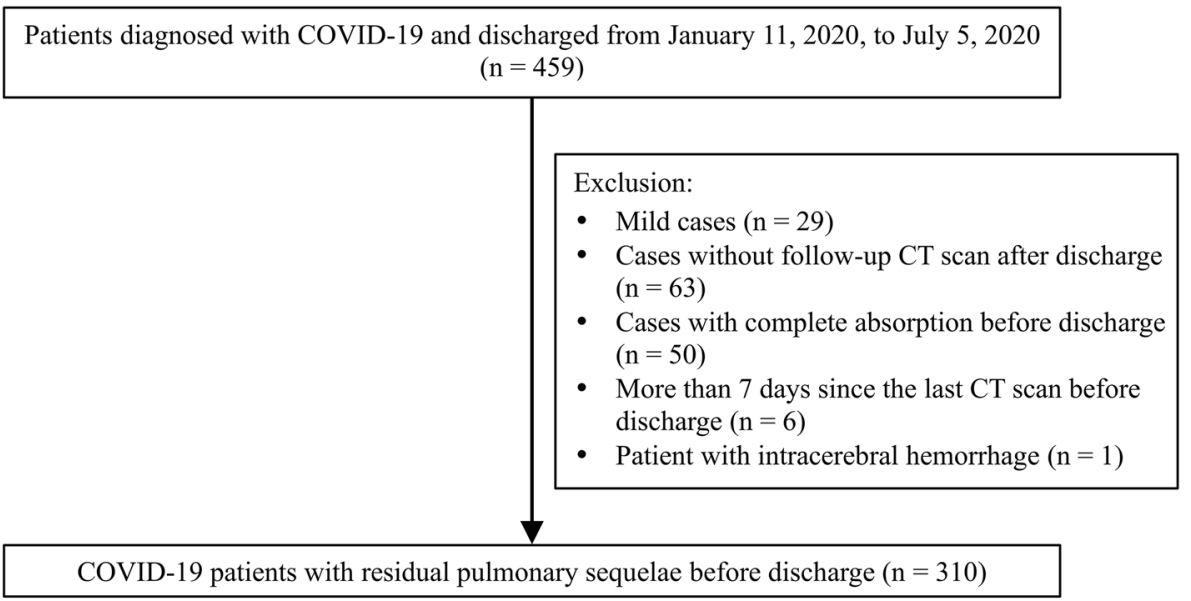

b

T0: 310

T1: 270

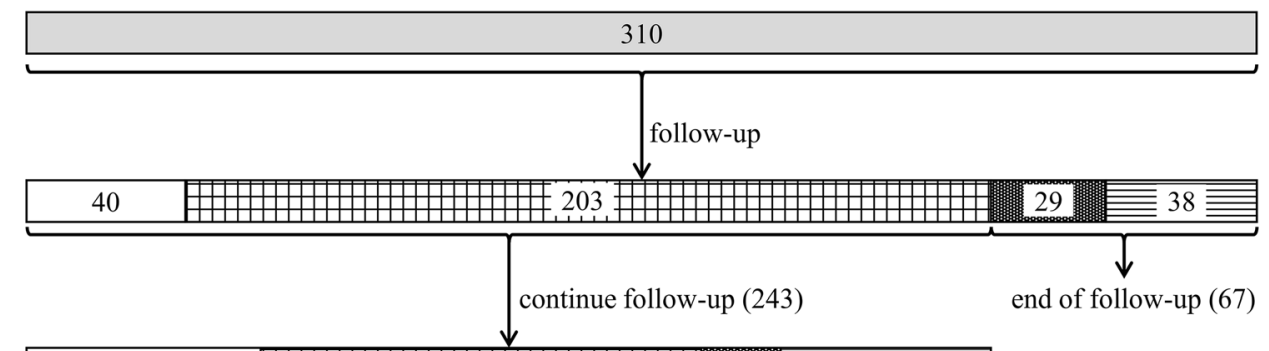

T2: 184

$59 \quad \mid \begin{array}{lll}59 \\ 111\end{array}$

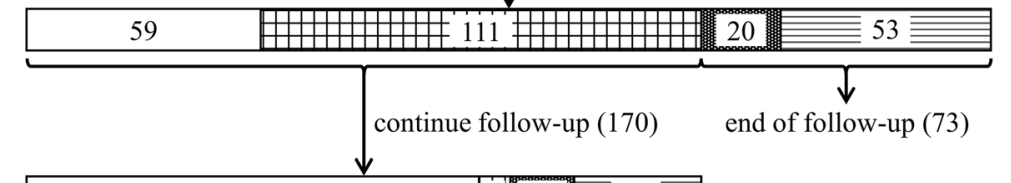

T3: 56

T4: 122

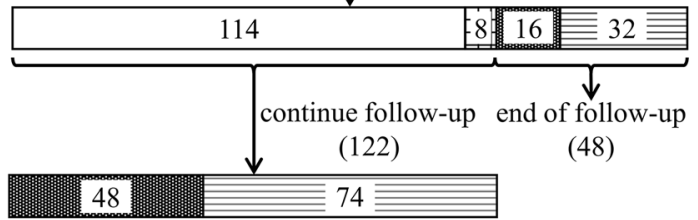

summary

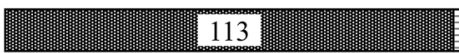

197

$\square$ total cases

complete absorption

residual pulmonary sequelae at last CT scan

without CT scan at corresponding period

巴performed CT scan at corresponding period and continued follow-up

Fig. 1 a Flow diagram of included and excluded patients. b Flow chart of the follow-up of all cases at each time point

periods were divided according to CT examination time. T0 was the number of days from the last pre-discharge CT scan to the time of discharge. T1 to T4 represent the follow-up periods after discharge-T1: 1-4 weeks, T2: 5-8 weeks, T3: 9-12 weeks, and T4: $>12$ weeks. These CT follow-up intervals were not strictly based on a 1-month follow-up in our study, but mainly on a comprehensive assessment by the clinicians. Therefore, we used an empirical method to divide postdischarge CT follow-up into stages $\mathrm{T} 1-\mathrm{T} 4$. The flowchart in
Figure $1 \mathrm{~b}$ illustrates the details of the follow-up at different time periods.

\section{CT scanning protocol}

Different CT scanners were used: AquilionTSX-101A (Toshiba Medical Systems), Ingenuity FIex (Philips Medical Systems), UCT760 (United Imaging Healthcare), and 
ANATOM 32 Fit (Anke Company). The reconstruction thickness ranged from 0.625 to $2 \mathrm{~mm}$.

\section{Interpretation of CT findings}

All CT images were independently reviewed by two radiologists (C. P. and J. Z.), and another senior radiologist (L. W., > 30 years' experience) made the final decision in cases of disagreement. The following imaging characteristics were evaluated:

1. Absorption of lesions: Complete absorption on radiological images (no lesions associated with COVID-19, Fig. 2c) and only residual fibrosis-like findings (residual fibrosis-like findings on $\mathrm{CT}$, but without any GGO or $\mathrm{CO})$.

2. Density: GGO, CO, and GGO with CO. GGOs were further divided into three sub-types as follows: (1) GGOs superimposed on irregular linear opacities (Fig. 3a): irregular linear opacities [12] were characterized as short and thin lines $(1-3 \mathrm{~mm})$ that were too small to be seen as a parenchymal band (described below), and the linear opacity was not caused by thickening of the interlobular septal and interlobular interstitium. (2) GGOs superimposed on reticulation: the reticulation [13] was characteristic of intralobular lines (Fig. 3a) or interlobular septal thickening (Fig. 3c) and was formed by these lines crossing each other. (3) Pure GGOs (pGGOs): no lesions were superimposed on GGOs (Fig. 3e).

3. Distribution and location: Peripheral (involving only the outer third of the lung), central (involving only the central two-thirds of the lung), or diffuse (involving both peripheral and central regions) [7]. Lung lobe involvement was recorded.

4. Fibrosis-like findings: The following fibrosis-like findings were evaluated in each CT scan. A parenchymal band $[12,13]$ refers to a linear opacity with a thickness of several millimeters and a length of up to $5 \mathrm{~cm}$ that crosses the pulmonary parenchyma and is often observed in the periphery (Fig. 4a, d). It often causes thickness and retraction of the contiguous pleura and causes distortion of the bronchial-vascular bundle and lung parenchyma. Bronchial dilatation (Fig. 4h, i) refers to a macroscopic change in the bronchus [14]. Irregular interface $[9,15,16]$ refers to an irregular or spiculated appearance of the pleural (Fig. 2d, e), bronchovascular (Fig. 4h, i), or mediastinal (Fig. 2df) surfaces. The radiologists also observed whether the a

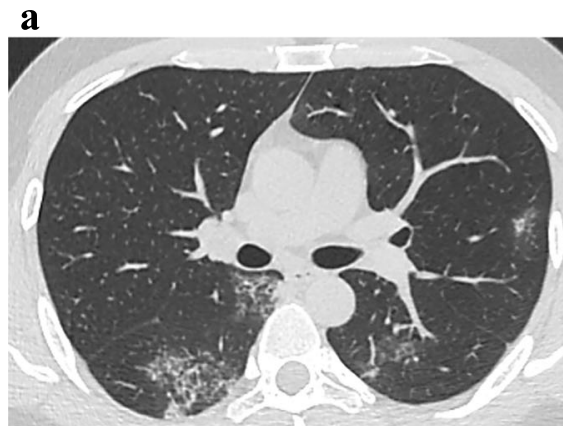

b

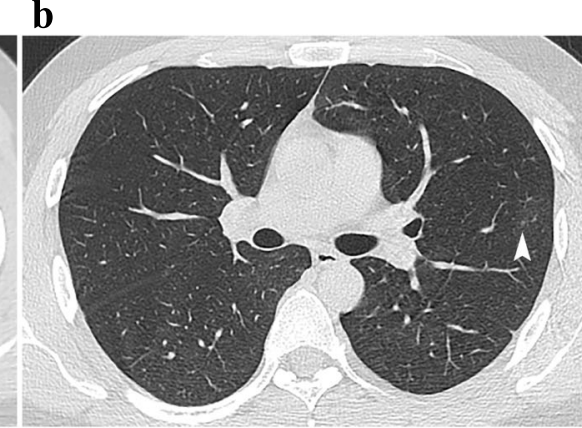

d
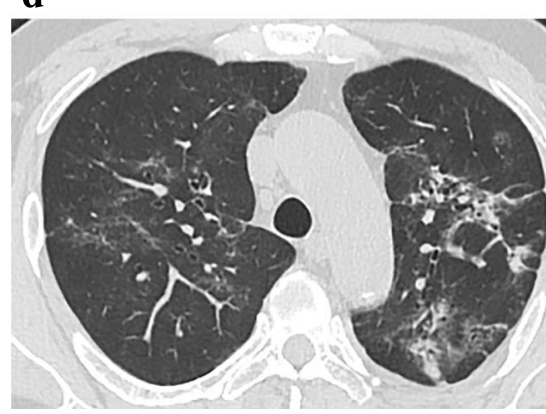

e

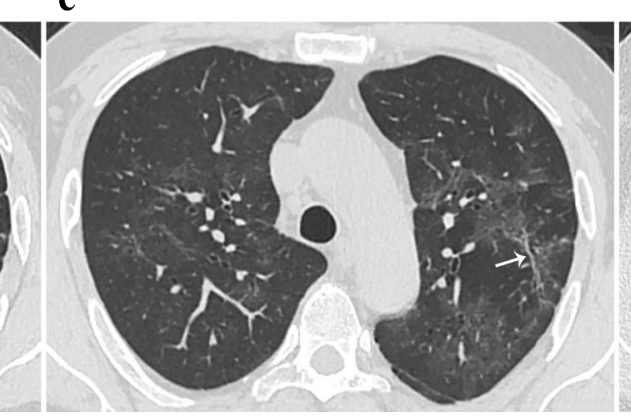

c

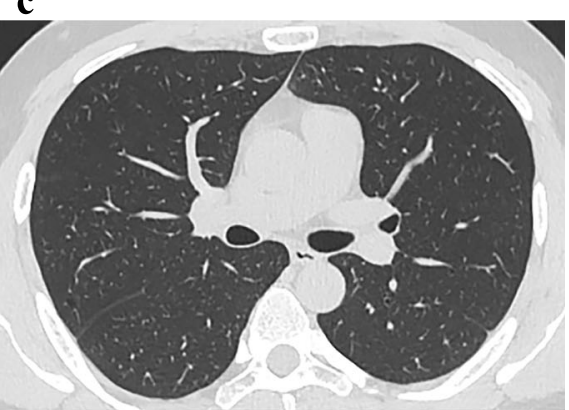

f

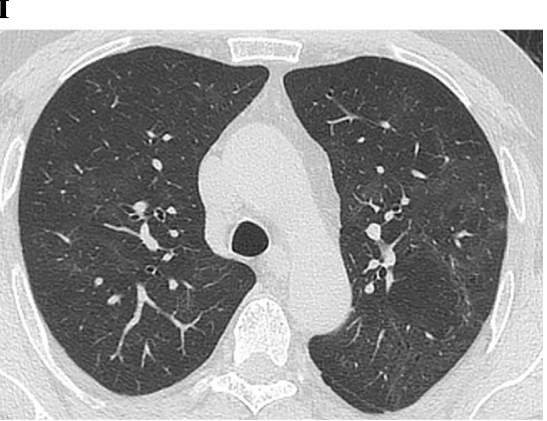

Fig. 2 a-c CT images of a 34-year-old male moderate patient at T0 (a), T1 (b), and T2 (c), showing the ground-glass opacities (GGOs), consolidations, and reticulation were gradually absorbed, until complete radiological absorption by T2. Pure GGO (pGGO) is seen in b (arrowhead). d- f CT images of a 56-year-old male critical patient at T0 (d), T2 (e), and T4 (f), reveal gradual absorption of lesions, but pGGO persisted in both lungs at T4. $\mathbf{d}-\mathbf{f}$ Irregular interfaces are found in the mediastinum. Irregular linear opacity is seen in $\mathbf{e}$ (arrow) 


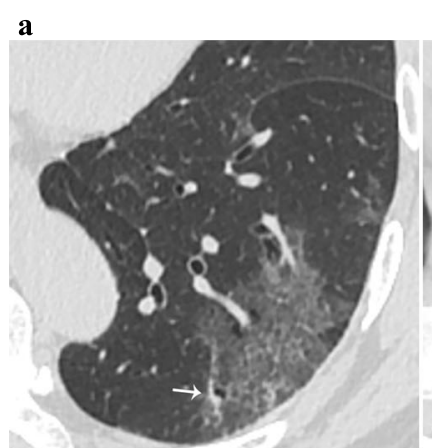

b$$
\text { c }
$$

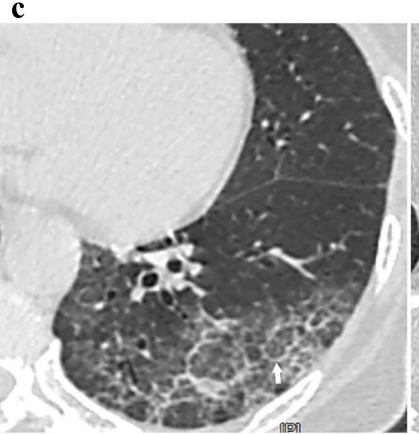

d
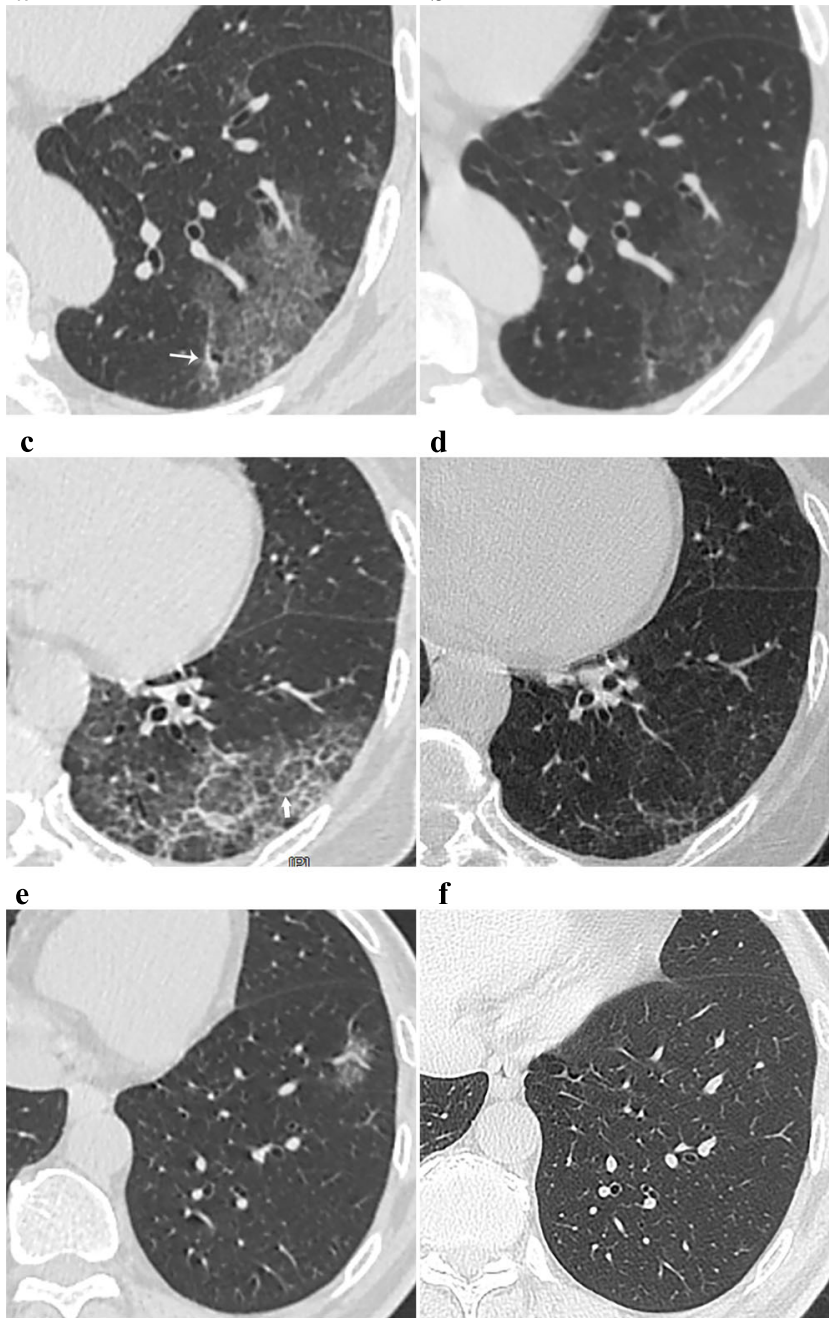

f

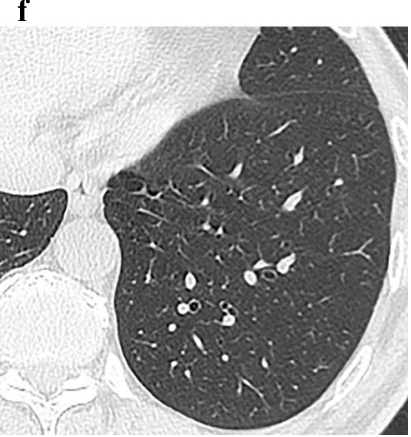

Fig. 3 Longitudinal variations in ground-glass opacities (GGOs). Patient 1 (a, b). a GGOs superimposed on fine reticulation at T0, which are caused by intralobular lines; irregular linear opacity was also found (thin arrow); b residual GGO and irregular linear opacity at T1. Patient 2 (c, d). c GGOs superimposed on coarse reticulation at T0 (thick arrow: interlobular septal thickening); $\mathbf{d}$ residual minimal GGO and thinner interlobular septal thickening at T2. Patient $3(\mathbf{e}, \mathbf{f})$. e Pure GGO at T0; f complete absorption at $\mathrm{T} 4$

irregular interface existed independently from other fibrosis-like findings.

\section{Statistical analysis}

SPSS software (SPSS 24.0, IBM Corporation) was used for all data analyses. Continuous variables are presented as medians (interquartile range [IQR]), and the Kruskal-Wallis test was used to compare continuous variables between the two groups. Categorical variables are presented as numbers (percentages) and were compared using Pearson's $\chi^{2}$ or the Fisher test. A $p$ value $<0.05$ was deemed statistically significant.

\section{Results}

\section{Comparison of basic clinical features}

The number of days for the T0, T1, T2, T3, and T4 periods were 1 (IQR: 1.0, 3.0), 17.0 (IQR: 16.0, 19.0), 33.0 (IQR: 31.0, 38.0), 71.0 (IQR: 61.0, 76.8), and 92.0 (IQR: 90.0, 100.0) days, respectively. Other clinical features are summarized in Table 1.

\section{Cumulative percentage of complete absorption}

The cumulative percentages of complete absorption in the severe and non-severe groups were $0(0 \%), 2(2.4 \%), 3(3.7 \%)$, and 10 (12.2\%); and 29 (12.7\%), 47 (20.6\%), 62 (27.2\%), and 103 (45.2\%), respectively, at $\mathrm{T} 1$ to $\mathrm{T} 4$ (Table 2).

\section{Cumulative rate of absorption of fibrosis-like findings}

During the study period, 172 patients, including 70 (85.4\%) severe/critical patients and $102(44.7 \%)$ moderate patients, showed fibrosis-like findings, which was present at $\mathrm{T} 0$ in 170 patients, and was found after discharge in the remaining two patients. The cumulative rate of absorption of fibrosis-like findings in the severe and non-severe groups was $3(4.3 \%), 11$ (15.7\%), 12 (17.1\%), and 17 (24.3\%); and 32 (31.4\%), 40 $(39.2 \%), 42(41.2 \%)$, and $53(52.0 \%)$, respectively, from T1 to T4 (Table 2).

\section{Comparison and longitudinal variations of lesion absorption}

The trend chart shows that the percentage of complete absorption gradually increased with time in both groups, and this percentage increased more obviously in the non-severe group than in the severe group (Fig. 5a). In terms of the proportion of patients with complete absorption, the first significant increase in the non-severe group was observed at T1 (T0 [0/228, 0\%] vs. T1 [29/203, 14.3\%], $p<0.001)$; however, such a significant increase in the severe group was observed at T4 (T0 $[0 / 82,0 \%]$ vs. T4 $[7 / 38,18.4 \%], p<0.001)$.

The presence of only residual fibrosis-like findings was observed in the non-severe group, and the incidence from T0 to T4 was $3(1.3 \%), 3(1.5 \%), 1(0.8 \%), 0(0 \%)$, and $7(8.3 \%)$, respectively. 
Fig. 4 Longitudinal variations in fibrosis-like findings. Patient 1 $(\mathbf{a}-\mathbf{c})$. Parenchymal band is gradually absorbed from T0 (a) through T1 (b), to T2 (c). Patient $2(\mathbf{d}-\mathbf{f})$. Parenchymal band persists at $\mathrm{T} 0$ (d) through $\mathrm{T} 2$ (e) to T4 (f). Patient 3 (g-i). g The initial computed tomography scan shows no bronchial dilatation in the right lower lobe. A typical bronchial dilatation is present at $\mathrm{T} 0$ (h) and T1 (i). Irregular interface of the bronchus is displayed (arrowhead) and a typical pattern of irregular linear opacities is also found in the periphery $(\mathbf{h}, \mathbf{i})$

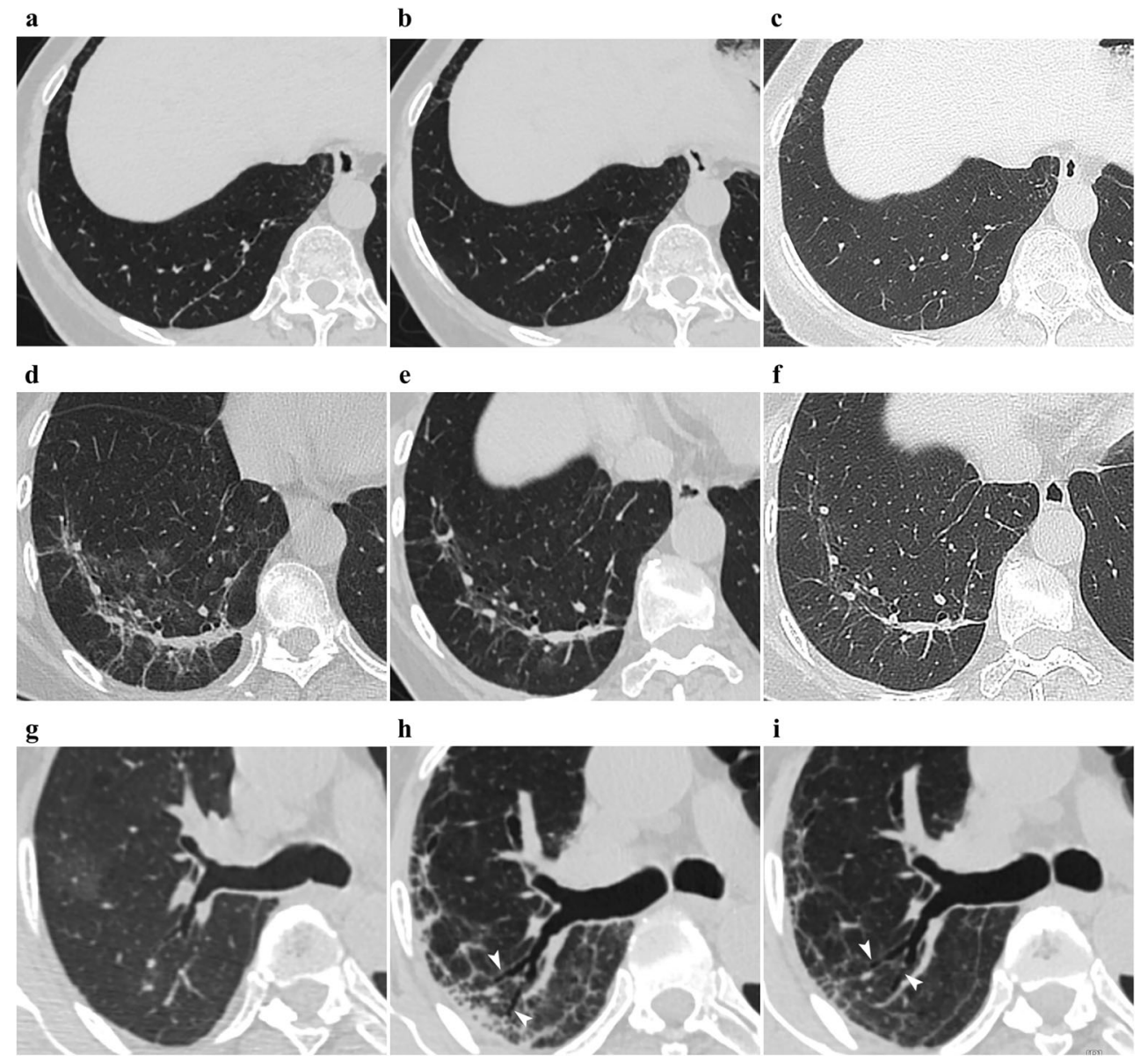

\begin{tabular}{lccrr}
\hline Characteristics & Severe, $n=82$ & Non-severe, $n=228$ & Total, $N=310$ & $p$ value \\
\hline Sex, $n(\%)$ & & & & 0.008 \\
Male & $51(62.2)$ & $103(45.2)$ & $154(49.7)$ & \\
Female & $31(37.8)$ & $125(54.8)$ & $156(50.3)$ & \\
Age (year, IQR) & $58.5(49.5,64.0)$ & $48.0(36.0,58.0)$ & $51(31.8,61.0)$ & $<0.001$ \\
Length of & $28.5(21.0,38.0)$ & $19.0(15.0,25.0)$ & $21.0(16.0,28.0)$ & $<0.001$ \\
$\quad$ hospitalization (day) & $25(30.5)$ & $26(11.4)$ & $51(16.5)$ & $<0.001$ \\
Underlying diseases, $n(\%)$ & $2(2.4)$ & $1(0.4)$ & $3(1.0)$ & 0.172 \\
Emphysema & $3(3.7)$ & $2(0.9)$ & $5(1.6)$ & 0.118 \\
Chronic bronchitis & $1(1.2)$ & $3(1.3)$ & $4(1.3)$ & 1.000 \\
OTB & $2(2.4)$ & $0(0)$ & $2(0.6)$ & 0.069 \\
Advanced ILD & $17(20.7 \%)$ & $16(7.0)$ & $33(10.6)$ & $<0.001$ \\
Hypertension & $7(8.5)$ & $4(1.8)$ & $11(3.5)$ & 0.004 \\
Diabetes & $6(7.3)$ & $2(0.9)$ & $8(2.6)$ & 0.002 \\
Coronary disease & & &
\end{tabular}

ILD, interstitial lung disease; IQR, interquartile range; OTB, obsolete pulmonary tuberculosis 
Table 2 Cumulative values of complete absorption and absorption of fibrosis-like findings in the severe and non-severe disease groups

\begin{tabular}{|c|c|c|c|c|c|c|}
\hline \multirow[t]{2}{*}{ Time period, $n(\%)$} & \multicolumn{3}{|c|}{$\begin{array}{l}\text { Cumulative values of complete } \\
\text { absorption }\end{array}$} & \multicolumn{3}{|c|}{$\begin{array}{l}\text { Cumulative values of absorption of fibrosis } \\
\text { like findings }\end{array}$} \\
\hline & $\begin{array}{l}\text { Severe, } \\
n=82\end{array}$ & $\begin{array}{l}\text { Non-severe, } \\
n=228\end{array}$ & $\begin{array}{l}\text { Total, } \\
N=310\end{array}$ & $\begin{array}{l}\text { Severe, } \\
n=70\end{array}$ & $\begin{array}{l}\text { Non-severe, } \\
n=102\end{array}$ & $\begin{array}{l}\text { Total, } \\
N=172\end{array}$ \\
\hline $\mathrm{T} 1$ & $0(0)$ & $29(12.7)$ & $29(9.4)$ & $3(4.3)$ & $32(31.4)$ & $35(20.3)$ \\
\hline $\mathrm{T} 2$ & $2(2.4)$ & $47(20.6)$ & $49(15.8)$ & $11(15.7)$ & $40(39.2)$ & $51(29.7)$ \\
\hline $\mathrm{T} 3$ & $3(3.7)$ & $62(27.2)$ & $65(21.0)$ & $12(17.1)$ & $42(41.2)$ & $54(31.4)$ \\
\hline $\mathrm{T} 4$ & $10(12.2)$ & $103(45.2)$ & $113(36.5)$ & $17(24.3)$ & $53(52.0)$ & $70(40.7)$ \\
\hline
\end{tabular}

T1, 1-4 weeks after discharge; T2, 5-8 weeks after discharge; T3, 9-12 weeks after discharge; T4, > 12 weeks after discharge

\section{Comparison and longitudinal variations in GGOs and COs}

The CT findings are compared between the two groups in Table 3. The frequency of GGO with $\mathrm{CO}$ in both groups showed a significant reduction at $\mathrm{T} 1$ (severe group: T0 [40/ $82,48.8 \%]$ vs. T1 [9/67, 13.4\%], $p<0.001$; non-severe group: T0 [31/228, $13.6 \%]$ vs. T1 [3/203, $1.5 \%], p<0.001)$. The frequency of GGO with $\mathrm{CO}$ in both groups remained stable from T2 to T4 (Fig. 5b).

The trend chart showed that the percentage of GGOs decreased gradually in the non-severe group, particularly at $\mathrm{T} 3$ (T0 [194/228, 85.1\%] vs. T3 [23/38, 60.5\%], $p<0.001$ ), while the severe group demonstrated a trend for an increase at T1 (T0 $[42 / 82,51.2 \%]$ vs. T1 $[58 / 67,86.6 \%], p<0.001)$ and then decreased at T4 (T2 [53/58, 91.4\%] vs. T4 [28/38, 73.7\%], $p=0.02$ ) (Fig. 5c). The most common GGO subtype in the severe group was GGOs superimposed on irregular linear opacities, the prevalence of which was significantly higher than in the non-severe group at each time period after discharge $(p<0.001$ [T1 to T4]). From T1 to T3, GGOs with superimposed reticulation was more common in the severe than in the non-severe group ( $p<0.001$ [T1], $p=0.002$ [T2], and $p=0.008$ [T3], respectively). The percentage of GGOs superimposed on irregular linear opacities and reticulation was immediately stabilized after discharge (Fig. 5d, e). pGGO was the predominant pattern in the non-severe group from $\mathrm{T} 0$ to $\mathrm{T} 3$.

At different time periods, the most common patterns of distribution in the severe and non-severe groups were diffuse and peripheral, respectively. From T0 to T3, the percentage of diffuse distribution gradually decreased, and the percentage of peripheral distribution gradually increased in the severe group (Fig. 6a).

The trend charts show that the GGOs or COs in each lobe gradually absorbed over time (Fig. $5 \mathrm{~g}-\mathrm{k}$ ). The absorption trend in the lower lobes in the severe group was more gradual (Fig. $5 \mathrm{i}, \mathrm{k})$. Compared to the non-severe group, a marked absorption trend in the severe group in the lower lobes was observed at T3 and T4 (right lower lobe: T0 [82/82, 100\%] vs. T3 [16/18, $88.9 \%], p=0.031$; left lower lobe: T0 [80/82, 97.6\%] vs. T4 $[28 / 38,73.7 \%], p<0.001)$. The total number of affected lobes was greater in the severe than in the non-severe group at each time point (all $p<0.001$, Table 3 and Fig. $6 \mathrm{~b}$ ).

\section{Comparison and longitudinal variations in fibrosis- like findings}

The percentage of patients with fibrosis-like findings, parenchymal band, and bronchial dilatation was significantly higher in the severe than in the non-severe group at each time point (all $p<0.05$, Table 3 ). The proportions of irregular interfaces and irregular pleural interfaces were significantly higher in the severe than in the non-severe group at $\mathrm{T} 0, \mathrm{~T} 1, \mathrm{~T} 2$, and $\mathrm{T} 4$ (all $p<0.05$, Table 3). Irregular bronchovascular interfaces were more common in the severe than in the non-severe group at T0 and T1 (all $p<0.05$, Table 3). Irregular mediastinal interfaces were very rare in both groups, without significant differences.

The trend charts for fibrosis-like findings were divided into two patterns (Fig. 51-r). Pattern 1: The percentage rapidly decreased at T1 or T2 (percentage: T0 vs. T1/T2, $p<0.05$ ) and then remained stable, including fibrosis-like findings, parenchymal band, irregular interfaces, and irregular pleural interfaces in both groups. Pattern 2: The percentage remained stable over time, including bronchial dilatation and irregular bronchovascular and mediastinal interfaces in both groups.

Cumulatively, 5/70 (7.1\%) patients showed irregular interfaces but no other fibrosis-like findings in the severe group during the study, compared to $3 / 102(2.9 \%)$ patients in the non-severe group.

\section{Discussion}

In this short-term follow-up study, serial CT scans were used to compare pulmonary damage and to systematically evaluate 
the changing trends in pulmonary sequelae in severe and nonsevere COVID-19 patients. The cumulative number of cases showing complete absorption of lung changes in the severe and non-severe groups at $\mathrm{T} 4$ showed superior pulmonary repair in the non-severe group compared to the repair in the severe group. Therefore, early intervention to avoid progression of mild/moderate disease to severe/critical disease is essential to reduce pulmonary sequelae. Additionally, for patients with fibrosis-like findings, the cumulative number of cases showing absorption in the severe and non-severe groups confirmed that partial fibrosis-like findings were absorbed after a short-term follow-up [17]. Furthermore, in a few patients, fibrosis-like findings without any GGO or CO remained, indicating that the absorption of fibrosis-like findings and $\mathrm{GGO} /$ $\mathrm{CO}$ was heterochronous.

The percentage of GGO with CO maintained similarly low levels between the two groups after the rapid absorption of $\mathrm{CO}$ by T1 (Fig. 5b). Liu et al [10] showed that only $2.0 \%$ of moderate COVID-19 patients exhibited persistent $\mathrm{CO}$ at the fourth week after discharge, similar to our findings. Moreover, we showed this to be true for severe/critical COVID-19 patients.

The incidence of GGOs in the non-severe group was markedly reduced from $85.1 \%$ at $\mathrm{T} 0$ to $41.7 \%$ at $\mathrm{T} 4$; however, the incidence in the severe group increased from $51.2 \%$ at $\mathrm{T} 0$ to $91.4 \%$ at $\mathrm{T} 2$, and then decreased to $73.7 \%$ at $\mathrm{T} 4$. The other most marked difference in the severe group was the proportion of GGO that remained high at $\mathrm{T} 4$. These results indicated that GGOs were absorbed slowly and persisted for a relatively long time in the severe group. Of note, the tendency for GGO to be superimposed on irregular linear opacities or reticulation remained stable after discharge. Irregular linear opacities and reticulation could develop into fibrotic lesions [18-20]; moreover, reticulation has been found to remain for as long as 7 years in SARS survivors [21]. Therefore, irregular linear opacities/reticulation may be an important factor in the slow absorption seen in the severe group.

In general, the absorption of GGOs and $\mathrm{CO}$ in the severe group has certain characteristics. First, from T0 to T3, the proportion of diffuse distribution in the severe group gradually decreased, accompanied by a simultaneous increase in the proportion of peripheral distribution, implying a trend for central absorption first and peripheral absorption later. Second, the absorption trend in both lower lobes was more stable than that in other lobes. Third, both lower lobes began to show significant absorption at $\mathrm{T} 3$ or $\mathrm{T} 4$ in the severe group. The most likely reason for this differential absorption pattern was that the lower lobes and lung periphery were most frequently involved in this disease [22,23]. Additionally, the influence of advanced age and underlying diseases on patients with COVID-19 cannot be ignored [24].

Postviral lung damage and fibrosis can lead to a decline in lung function and quality of life $[25,26]$. In our study, 29.1\%
(59/203) of patients in the non-severe group showed fibrosislike findings at T1, similar to a previous study [9]. What is more concerning is that the frequency of fibrosis-like findings was relatively higher in the severe disease group.

Parenchymal band is the most common among fibrosis-like findings and appears as early as the second week after onset $[8$, 27]. They usually represent atelectasis or advanced pulmonary fibrosis [12]. A significant downward trend in the frequency of parenchymal bands was observed at $\mathrm{T} 1$ or T2 (Fig. $5 \mathrm{~m}$ ), suggesting that the absorbed parenchymal bands may represent temporary atelectasis. The risk of fibrosis must be considered in patients with persistent parenchymal bands. Compared to patients with moderate disease, bronchial dilatation in patients with severe/critical disease persisted at a relatively stable medium level at all stages. Whether bronchial dilatation persists and eventually evolves into traction bronchiectasis is an important consideration, as traction bronchiectasis is associated with mortality $[28,29]$. Irregular interfaces rarely existed independently in this study, confirming that an irregular interface is a nonspecific $\mathrm{CT}$ finding usually accompanied by other more specific signs [16]. Furthermore, irregular interfaces were observed most commonly in the pleura, while irregular interfaces were rare in the bronchovascular bundle and mediastinum. This appears to be related to the distribution characteristics of COVID19 pneumonia [30].

There were some limitations to the present study. First, there were many patients for whom the outcome and final prognosis were not observed. Moreover, the follow-up time was too short to confirm that the residual fibrosis-like findings represented mature fibrosis. Consequently, studies with longterm follow-ups are necessary. Second, the impact of residual pulmonary sequelae on the daily life of patients and the severity of lung injury was not evaluated in detail. Future clinical multivariate analyses, including lung function and activity tolerance, are needed. Third, there was no pathological evidence to assess the fibrosis-like findings.

In conclusion, this study demonstrated short-term patterns and changing trends of pulmonary sequelae (GGO, $\mathrm{CO}$, and fibrosis-like findings) in COVID-19 patients after discharge. Lung sequelae were more serious and recovery was slower in patients with severe/critical disease than in patients with moderate disease. Fibrosis-like findings were completely absorbed after the short-term follow-up in more than $20 \%$ of patients with severe/critical disease and more than $50 \%$ of patients with moderate disease.

Fig. 5 Longitudinal variations of pulmonary sequelae (including groundglass opacities, consolidations, and fibrosis-like findings) in the severe and non-severe group, from T0 to T4. The symbols $(*, \dagger, t)$ indicate significant differences between the two groups at the corresponding time points $(* p<0.001, \dagger p<0.01, \ddagger p<0.05)$. CO, consolidation; GGO, ground-glass opacity 

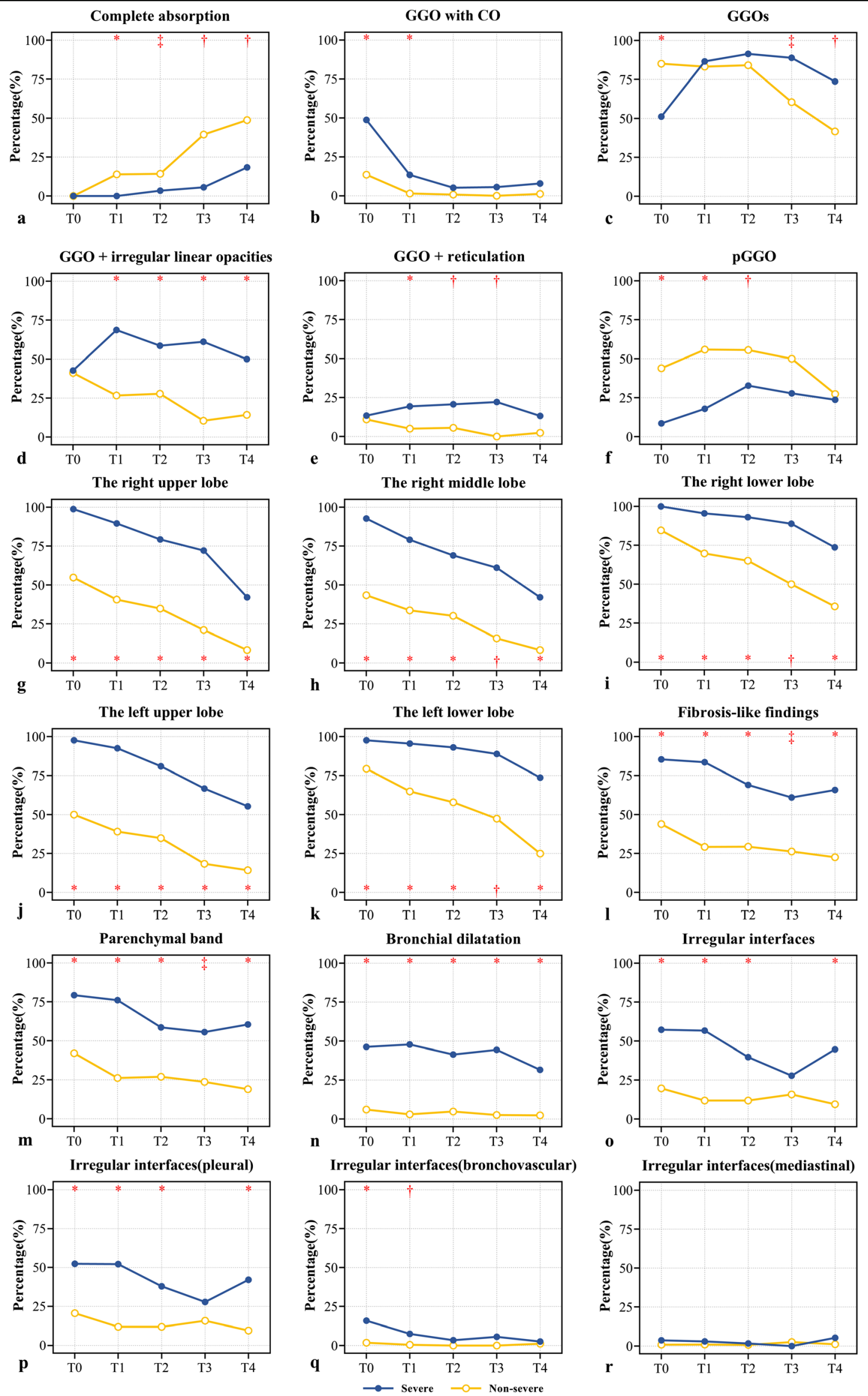


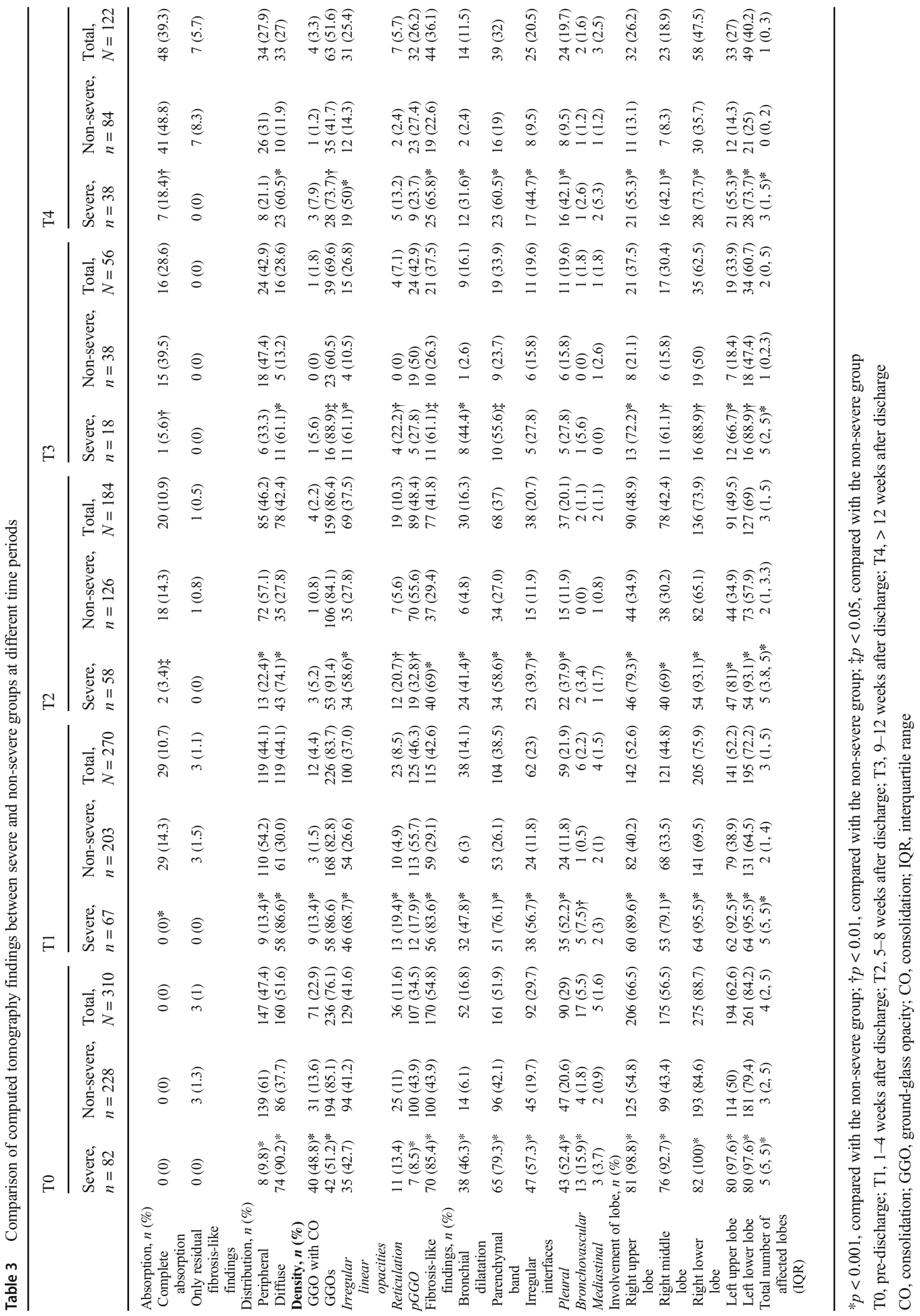




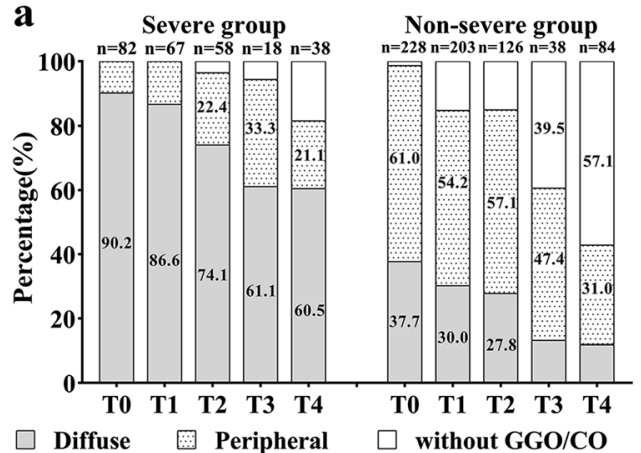

Fig. 6 a Distribution of ground-glass opacities and consolidations. Categories with percentage $\geq 20 \%$ were shown. Without GGO/CO refers to the pattern of complete absorption or only residual fibrosis. $\mathbf{b}$ The total

Supplementary Information The online version contains supplementary material available at https://doi.org/10.1007/s00330-021-07799-9.

Acknowledgements We thank Guangping Zheng for assistance with data collection.

Funding This study received funding from the Sanming Project of Medicine in Shenzhen (fund No. SZSM201612053).

\section{Declarations}

Guarantor The scientific guarantor of this publication is Lifei Wang.

Conflict of interest The authors of this manuscript declare no relationships with any companies whose products or services may be related to the subject matter of the article.

Statistics and biometry No complex statistical methods were necessary for this paper.

Informed consent Written informed consent was waived by the Institutional Review Board.

Ethical approval Ethical Approval was obtained from the Ethics Committee of the Shenzhen Third People's Hospital (Certification No. 2020-227).

\section{Methodology}

- retrospective

- comparative study

- single-center study

\section{References}

1. Li Q, Guan X, Wu P et al (2020) Early transmission dynamics in Wuhan, China, of novel coronavirus-infected pneumonia. N Engl J Med 382:1199-1207. https://doi.org/10.1056/NEJMoa2001316 b

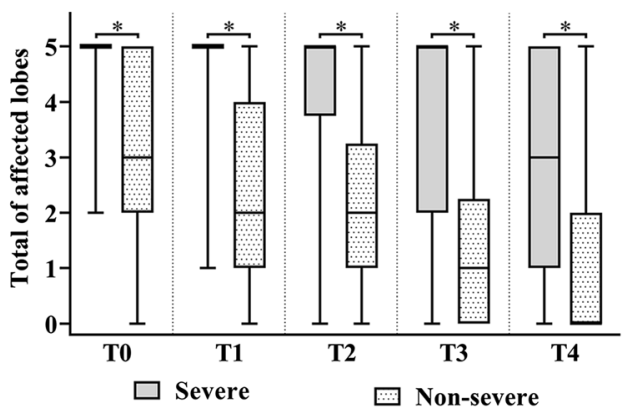

number of affected lobes in the two groups. The symbol (*) indicates a significant difference between the two groups at the corresponding time point $(* p<0.001)$. CO, consolidation; GGO, ground-glass opacity

2. Zhu N, Zhang D, Wang W et al (2020) A novel coronavirus from patients with pneumonia in China, 2019. N Engl J Med 382:727733. https://doi.org/10.1056/NEJMoa2001017

3. World Health Organization (2020) Coronavirus disease 2019 (COVID-19) situation report. 203. World Health Organization, Geneva. Available via https://www.who.int/docs/default-source/ coronaviruse/situation-reports/20200810-covid-19-sitrep-203.pdf? sfvrsn=aa050308 4. Accessed 10 Aug 2020

4. Li X, Zeng W, Li X et al (2020) CT imaging changes of corona virus disease 2019(COVID-19): a multi-center study in Southwest China. J Transl Med 18:154. https://doi.org/10.1186/s12967-02002324-w

5. Zheng Z, Yao Z, Wu K, Zheng J (2020) Patient follow-up after discharge after COVID-19 pneumonia: considerations for infectious control. J Med Virol. https://doi.org/10.1002/jmv.25994

6. Du S, Gao S, Huang G et al (2020) Chest lesion CT radiological features and quantitative analysis in RT-PCR turned negative and clinical symptoms resolved COVID-19 patients. Quant Imaging Med Surg 10:1307-1317. https://doi.org/10.21037/qims-20-531

7. Wang Y, Dong C, Hu Y et al (2020) Temporal changes of CT findings in 90 patients with COVID-19 pneumonia: a longitudinal study. Radiology. 296:E55-E64. https://doi.org/10.1148/radiol. 2020200843

8. Yu M, Liu Y, Xu D, Zhang R, Lan L, Xu H (2020) Prediction of the development of pulmonary fibrosis using serial thin-section CT and clinical features in patients discharged after treatment for COVID19 pneumonia. Korean J Radiol 21:746-755. https://doi.org/10. 3348/kjr.2020.0215

9. Wei J, Yang H, Lei P et al (2020) Analysis of thin-section CT in patients with coronavirus disease (COVID-19) after hospital discharge. J Xray Sci Technol 28:383-389. https://doi.org/10.3233/ XST-200685

10. Liu C, Ye L, Xia R et al (2020) Chest CT and clinical follow-up of discharged patients with COVID-19 in Wenzhou City, Zhejiang, China. Ann Am Thorac Soc 17:1231-1237. https://doi.org/10. 1513/AnnalsATS.202004-324OC

11. Chinese National Health Committee (2020) Diagnosis and treatment of COVID-19 pneumonia. (trial seventh edition) Available via http://www.nhc.gov.cn/yzygj/s 7653 p/202003/ 46c9294a7dfe4cef80dc7f5912eb1989.shtml. Accessed 3 Mar 2020

12. Verschakelen JA, De Wever W (2018) Linear pattern. In: Computed Tomography of the Lung. Medical Radiology. Springer, Berlin, Heidelberg. https://doi.org/10.1007/978-3-64239518-5 7 
13. Hansell DM, Bankier AA, MacMahon H, McLoud TC, Müller NL, Remy J (2008) Fleischner Society: glossary of terms for thoracic imaging. Radiology. 246:697-722. https://doi.org/10.1148/radiol. 2462070712

14. Javidan-Nejad C, Bhalla S (2009) Bronchiectasis. Radiol Clin North Am 47:289-306. https://doi.org/10.1016/j.rcl.2008.11.006

15. Antonio GE, Wong KT, Hui DS et al (2003) Thin-section CT in patients with severe acute respiratory syndrome following hospital discharge: preliminary experience. Radiology. 228:810-815. https://doi.org/10.1148/radiol.2283030726

16. Webb R, Muller NL, Naidich DP (2015) High-resolution CT of the lung, 5th edn. Wolters Kluwer Publishers, Philadelphia

17. Liu D, Zhang W, Pan F et al (2020) The pulmonary sequalae in discharged patients with COVID-19: a short-term observational study. Respir Res 21:125. https://doi.org/10.1186/s12931-02001385-1

18. Lee JS, Lynch DA, Sharma S, Brown KK, Müller NL (2003) Organizing pneumonia: prognostic implication of high-resolution computed tomography features. J Comput Assist Tomogr 27:260 265. https://doi.org/10.1097/00004728-200303000-00027

19. Kim EY, Lee KS, Chung MP, Kwon OJ, Kim TS, Hwang JH (1999) Nonspecific interstitial pneumonia with fibrosis: serial high-resolution CT findings with functional correlation. AJR Am J Roentgenol 173:949-953. https://doi.org/10.2214/ajr.173.4. 10511155

20. Mai C, Verleden SE, McDonough JE et al (2017) Thin-section CT features of idiopathic pulmonary fibrosis correlated with micro-CT and histologic analysis. Radiology. 283:252-263. https://doi.org/ 10.1148/radiol.2016152362

21. Wu X, Dong D, Ma D (2016) Thin-section computed tomography manifestations during convalescence and long-term follow-up of patients with Severe Acute Respiratory Syndrome (SARS). Med Sci Monit 22:2793-2799. https://doi.org/10.12659/msm.896985

22. Li K, Wu J, Wu F et al (2020) The clinical and chest CT features associated with severe and critical COVID-19 pneumonia. Invest
Radiol 55:327-331. https://doi.org/10.1097/RLI. 0000000000000672

23. Emami A, Javanmardi F, Pirbonyeh N, Akbari A (2020) Prevalence of underlying diseases in hospitalized patients with COVID-19: a systematic review and meta-analysis. Arch Acad Emerg Med 8:e35

24. Clerkin KJ, Fried JA, Raikhelkar J et al (2020) COVID-19 and cardiovascular disease. Circulation. 141:1648-1655. https://doi. org/10.1161/CIRCULATIONAHA.120.046941

25. Batawi S, Tarazan N, Al-Raddadi R et al (2019) Quality of life reported by survivors after hospitalization for Middle East respiratory syndrome (MERS). Health Qual Life Outcomes 17:101. https://doi.org/10.1186/s12955-019-1165-2

26. Ngai JC, Ko FW, Ng SS, To KW, Tong M, Hui DS (2010) The long-term impact of severe acute respiratory syndrome on pulmonary function, exercise capacity and health status. Respirology. 15: 543-550. https://doi.org/10.1111/j.1440-1843.2010.01720.x

27. Pan F, Ye T, Sun P et al (2020) Time course of lung changes at chest CT during recovery from coronavirus disease 2019 (COVID19). Radiology. 295:715-721. https://doi.org/10.1148/radiol. 2020200370

28. Takei R, Arita M, Kumagai S et al (2019) Traction bronchiectasis on high-resolution computed tomography may predict fatal acute eosinophilic pneumonia. Respir Investig 57:67-72. https://doi.org/ 10.1016/j.resinv.2018.09.005

29. Hida T, Nishino M, Hino $T$ et al (2020) Traction bronchiectasis/ bronchiolectasis is associated with interstitial lung abnormality mortality. Eur J Radiol 129:109073. https://doi.org/10.1016/j. ejrad.2020.109073

30. Bao C, Liu X, Zhang H, Li Y, Liu J (2020) Coronavirus disease 2019 (COVID-19) CT findings: a systematic review and meta-analysis. J Am Coll Radiol 17:701-709. https://doi.org/10.1016/j.jacr. 2020.03.006

Publisher's note Springer Nature remains neutral with regard to jurisdictional claims in published maps and institutional affiliations. 